\title{
Progress and limits of PrP gene selection policy
}

\author{
Michael DAwson ${ }^{1 *}$, Richard C. MoorE ${ }^{2}$, Stephen C. BISHOP ${ }^{3}$ \\ ${ }^{1}$ National Scrapie Plan Administration Centre, Animal Health, Worcester, WR5 2SU, UK \\ ${ }^{2}$ Roslin Institute, Roslin, Midlothian, EH25 9PS, UK \\ ${ }^{3}$ Roslin Institute and Royal (Dick) School of Veterinary Studies, Roslin BioCentre, Midlothian, EH25 9PS, UK
}

(Received 4 September 2007; accepted 13 November 2007)

\begin{abstract}
Classical scrapie has proved to be a notoriously difficult disease to control due to a poor understanding of its natural history. The recognition of disease risk linkage to PrP genotype has offered the prospect of a disease control strategy, viz. genotyping and selective breeding, novel to veterinary medicine when first considered in the 1990s. The UK Spongiform Encephalopathy Advisory Committee recommended the exploitation of this approach in a voluntary, national programme to control classical scrapie and protect the public against food-borne exposure to bovine spongiform encephalopathy, should the national flock have been exposed via contaminated feed. The National Scrapie Plan for Great Britain was launched in 2001 and uptake has been widespread throughout the purebreeding sector of the sheep industry, with membership peaking at over 12000 flocks in 2006. A total of 700000 rams from 90 breeds have been genotyped. A comparison of ram lambs born in 2002 with those in 2006 shows evident changes in PrP genotype frequencies which are predicted to be associated with a reduction in disease risk. Various concerns have been raised regarding possible unintended consequences of widespread selection on $\operatorname{PrP}$ genotype, including impacts on other performance traits and possible effects on inbreeding and genetic diversity. To date, these concerns appear to be unfounded, as no consistent associations have been found with performance traits, nor are there likely to be any detectable impacts on inbreeding in mainstream breeds. Currently, semen banks have been implemented in Great Britain to store samples from animals of all common PrP genotypes, should these genotypes be required in the future. Various strategies to minimise future disease risks are discussed in the paper.
\end{abstract}

scrapie / genotyping / control / limitations / concerns

Table of contents

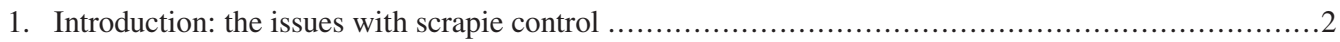

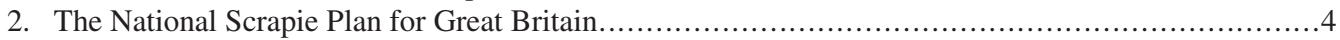

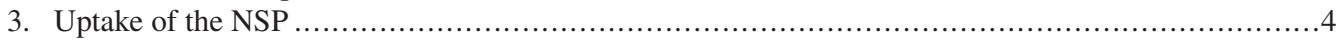

4. Impact of the Ram Genotyping Scheme on genotype profiles and allele frequency ..................... 4

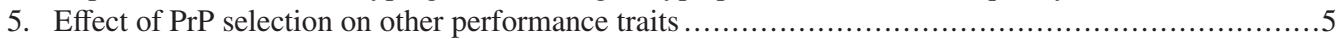

6. Effect of PrP selection on inbreeding and genetic diversity ..........................................

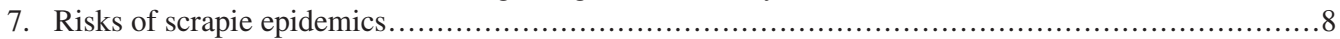

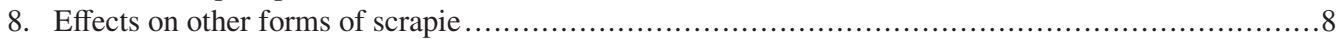

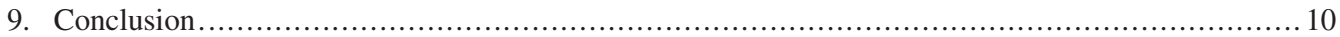

*Corresponding author: michael.dawson@ah.gsi.gov.uk 


\section{INTRODUCTION: THE ISSUES WITH SCRAPIE CONTROL}

Classical scrapie is a fatal neurodegenerative disease of sheep and goats that has been endemic in Britain for at least three centuries [29] and is also recognised in many of the sheep-rearing countries of the world: notable exceptions are Australia and New Zealand. It has been classified as a single disease entity within the group of diseases known as the transmissible spongiform encephalopathies (TSE), or prion diseases. However, the known spectrum of prion diseases of small ruminants has been extended recently with the recognition of previously undescribed variants, e.g., Nor 98 and atypical scrapie (AS) $[1,4,5,23,27,32]$.

Historically, classical scrapie has proved to be a difficult disease to control. A poor understanding of the aetiology, pathogenesis, and epidemiology has severely limited options for disease control. The failure to identify a conventional micro-organism as the aetiological agent has meant that the development of direct or indirect diagnostic assays for use in live sheep and goats has been a relatively barren area of applied research. Biopsy of accessible lymphoid tissue e.g., tonsil [33], third eyelid [28], and lymphoid tissue associated with rectal mucosa [16], followed by assay for prion protein do offer options for preclinical diagnosis and can be applied routinely to suspect single cases, or small groups of animals. However, cost, practicality, and welfare considerations inhibit widespread application at the flock level. Routinely, therefore, recognition of infected sheep is normally achieved after the emergence of clinical signs and using diagnostic assays on brain and tissue samples collected following post mortem examination. Restrictions on disease control options caused by the availability of limited diagnostics are further compounded by poor knowledge of the routes of transmission. Infected ewes may disseminate infection via uterine discharges, contaminating the lambing environment, more than once in a prolonged period of clinical latency: peak age incidence for scrapie is 24 years, but a significant minority of cases occur in older sheep.
The potential for PrP genotyping to control scrapie started to emerge in the 1990s when case control studies [20] demonstrated clear linkage between classical scrapie disease risk and PrP genotype in several breeds of sheep in Britain. The significant polymorphisms linked to disease risk are at codons 136 (alanine/valine), 154 (arginine/histidine), and 171 (glutamine/histidine/arginine) of the ovine PrP gene. Combinations of these polymorphisms lead to five commonly seen PrP haplotypes or alleles (Tab. I). PrP genotyping was made available to sheep farmers in Britain in the early 1990s to aid selection of rams for breeding. One of the first breeds to benefit was the Swaledale [10], a hill breed from the north of England with a history of endemic classical scrapie in which previous studies [9] had strongly indicated a genetic predisposition to experimental challenge.

The impetus to control disease at a national level increased dramatically from the mid1990s, for two main reasons: the emergence of variant CJD [40] and the demonstration that BSE could be transmitted to sheep by the oral route [15]. More farmers started to have their sheep PrP, or "scrapie", genotyped as they recognised the benefits that this novel tool presented. Tested rams of certain genotypes could be purchased with more confidence, knowing that there was little risk of them developing future classical scrapie disease, or increasing disease risk in their progeny. In the UK, an advisory group, the Scrapie Information Group, comprising government officials, scientists, veterinarians, and industry members, published guidelines on the use and interpretation of genotyping results [11]. The concern that the national flock might have been exposed to BSE via contaminated sheep feed prompted the Government's Spongiform Encephalopathy Advisory Committee to recommend a control programme based on PrP genotyping. Modelling studies by the Veterinary Laboratories Agency [2] showed that genotyping focussed on purebred ram lambs, followed by selective breeding, would have the greatest impact on disseminating classical scrapie resistance, so reducing the risk of classical scrapie in the national flock. Based on this 
Table I. The 15 PrP genotypes and their allocation to risk groups. Rams are awarded a certificate according to genotype. The use of types 1, 2 and 3 rams is not restricted. Type 4 and 5 rams are required to be culled or castrated.

\begin{tabular}{lll}
\hline Genotype & NSP Group & Associated scrapie risk \\
\hline ARR/ARR & 1 & Very low \\
\hline ARR/AHQ & 2 & Low \\
ARR/ARH & & \\
ARR/ARQ & & Moderate, especially in ARQ/ARQ \\
\hline AHQ/AHQ & 3 & \\
AHQ/ARH & & \\
AHQ/ARQ & & \\
ARH/ARH & & \\
ARH/ARQ & & Moderate \\
ARQ/ARQ & & High, especially in ARQ/VRQ and VRQ/VRQ \\
\hline ARR/VRQ & 4 & \\
\hline AHQ/VRQ & 5 & \\
ARH/VRQ & & \\
ARQ/VRQ & & \\
VRQ/VRQ & & \\
\hline
\end{tabular}

NSP: National Scrapie Plan.

modelling, the National Scrapie Plan (NSP) for Great Britain was launched in July 2001 with the twin aims of controlling classical scrapie and protecting the consumer from the exposure to BSE via sheep meat, should the national flock have become infected.

In addition to its adoption in the UK, the broad strategy of attempting to control classical scrapie based on PrP genotype selection has been adopted to a greater or lesser extent in several European Countries - notably The Netherlands from 1998, France, and Eire. Programmes have also been operated in North America. There has been considerable variation in scheme application. In terms of positive selection for the ARR allele, the Dutch scheme seems to have been the most aggressive: it was designed with the aim of producing 50000 ARR homozygote rams by 2004 to enable production of "safe" lamb for the consumer. In France, the strategy has been to target elite selection flocks. In Eire, the choice of animals to genotype has been left more to the discretion of the breeder.

European Commission Decision 2003/100/ EC provided for the introduction of EU-wide genotype-based breeding programmes on a voluntary basis from 2004 and then on a com- pulsory basis from 1 April 2005. However, the European Parliament and the Council of Ministers expressed reservations, in particular about the risk of adverse impacts on genetic diversity and on rare and locally adapted breeds. The requirement for all member states to adopt compulsory breeding programmes was therefore dropped. It was left to the discretion of each member state whether or not to pursue voluntary schemes.

The aim of this paper is to describe and discuss progress and limitations of selection on PrP genotype. As a case study it considers the extent to which selection on PrP genotype under the auspices of the NSP (www.defra.co.uk/nsp) has been successful in Great Britain, as measured by scheme uptake, numbers of rams tested and the resulting annual changes in genotype profiles and allele frequencies. It then briefly considers possible impacts on other performance traits, inbreeding, risks of classical scrapie epidemics, and the potential prevalence of other forms of scrapie. It also briefly describes measures that are currently being taken to safeguard against future risks, through the development of a semen archive containing semen straws from all major susceptible PrP genotypes. 


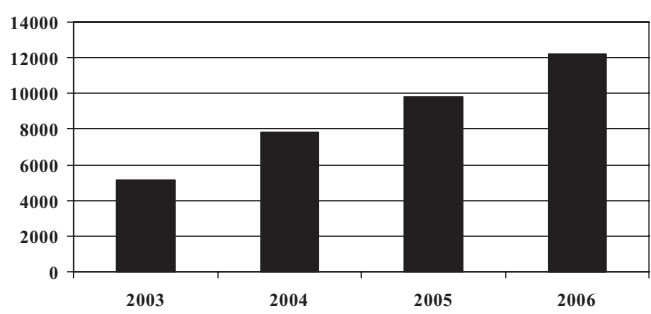

Figure 1. Annual progression in flock membership (number of flocks) of the National Scrapie Plan (NSP) Ram Genotyping Scheme.

\section{THE NATIONAL SCRAPIE PLAN FOR GREAT BRITAIN}

The Ram Genotyping Scheme (RGS) has been the main element of the NSP. This is a voluntary programme, introduced in 2001, with all costs - blood sampling, electronic identification, laboratory genotyping, supply of certificates, and scheme administration met by government. Members have been entitled to annual sampling visits at which all rams and ram lambs have been genotyped, irrespective of whether they are participating in structured breed improvement programmes. Each of the 15 PrP genotypes (i.e. pairings of the five alleles defined by the polymorphisms at codons 136, 154, and 171) have been assigned to one of five groups, according to the risk of disease in the individual and in first generation progeny (Tab. I) [39].The plan requires negative selection of the VRQ allele and encourages positive selection for the ARR allele. Rams with the VRQ allele have to be killed or castrated.

\section{UPTAKE OF THE NSP}

A productive working relationship was established from the outset with the National Sheep Association and the majority of its constituent breed societies. This cooperation resulted in widespread uptake of the RGS by sheep breeders, which is illustrated in Figure 1. The numbers of ram lambs tested annually are given at Figure 2. Given that the average working life of a ram in Britain is 4-5 years and there are estimated to be $400000 \mathrm{rams}$ used each year in Britain [30], it seems probable that the majority of working rams will have

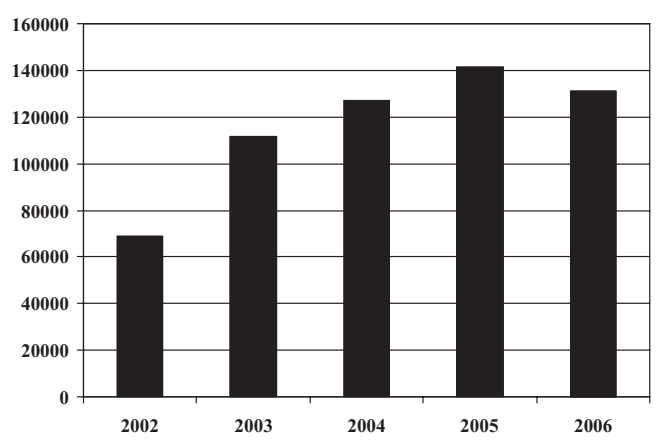

Figure 2. Numbers of ram lambs genotyped each year, from 2002 to 2006 .

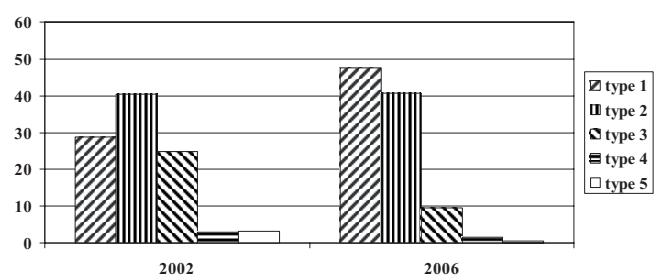

Figure 3. The frequency (\%) distribution of $\mathrm{Na}-$ tional Scrapie Plan (NSP) genotype groupings (see Tab. I) of ram lambs tested in the NSP for 2002 and 2006.

been genotyped by now. Flock membership of the scheme has steadily risen and peaked at over 12000 flocks, a figure estimated to represent $80 \%$ of flocks producing breeding rams. More than 700000 rams from 90 different breeds have been genotyped.

\section{IMPACT OF THE RAM GENOTYPING SCHEME ON GENOTYPE PROFILES AND ALLELE FREQUENCY}

To monitor the progress of the RGS, the genotype profiles and allele frequencies have been assessed for successive populations of ram lambs sampled each year and also for each of the major breeds [39]. Figure 3 illustrates the shift in genotype profile of all ram lambs that has been achieved from 2001 to 2006. Type 1 genotypes have increased from $28.8 \%$ to $47.6 \%$; type 2 genotypes have remained around the $40 \%$ level; type 3 genotypes have shown a reduction from $24.9 \%$ to $9.4 \%$, and for the VRQ-carrying genotypes, types 4 and 
Table II. Changes in allele frequencies, comparing the 2002 cohort of ram lambs with that of 2006 .

\begin{tabular}{cccc}
\hline Allele & 2002 & 2006 & Percentage change \\
\hline ARR & 50.4 & 68.8 & +36.5 \\
AHQ & 7.4 & 5.5 & -25.7 \\
ARH & 9.9 & 5.6 & -43.4 \\
ARQ & 29.2 & 18.9 & -35.3 \\
VRQ & 3.0 & 1.2 & -60.0 \\
\hline
\end{tabular}

5 , the reduction is from $5.9 \%$ to $2.3 \%$. The shift in allele frequencies is recorded in Table II. The changes recorded for the tested ram lambs have been: an increase in the frequency of the ARR allele from $50.4 \%$ to $68.8 \%$, a relative increase of $36.5 \%$; a reduction in the frequency of the ARQ allele from $29.2 \%$ to $18.9 \%$, a relative reduction of $35.3 \%$; a reduction in the frequency of the VRQ allele from $3 \%$ to $1.2 \%$, a relative reduction of $60 \%$. As the changes in allele frequencies will be progressively reflected in the ewes bred by these rams, it is to be expected that the risk of classical scrapie in the national flock will diminish, as described below.

In addition to the RGS, which has aimed to reduce disease risk across the national flock, control action has also been targeted at known affected flocks to reduce the risk of further disease transmission. Approximately 200 farmers with cases occurring between to 1998 and 2004 volunteered to attempt control based on genotyping, selective culling of atrisk genotypes and subsequent breeding with low risk genotypes. Furthermore, an additional 250 holdings with cases occurring since 2004 have been subject to the compulsory control measures - genotype and cull, or whole flock cull - as required by EC regulation 999/2001 as amended. In these 450 holdings, there has been as yet only a single case of further disease in a type 2 ewe in low-risk sheep selected for retention for further breeding.

EU TSE regulation 999/2001 as amended requires EU member states to conduct surveillance of fallen stock over 18 months of age and ewes over 18 months of age submitted to abattoirs for evidence of scrapie. In Great Britain there are positive signs from these surveys that the prevalence of infection is falling.
In 2003, the prevalence figures for the abattoir survey and fallen stock survey were $0.06 \%$ and $0.23 \%$, respectively. In 2006, these prevalences had fallen to $0.02 \%$ and $0.17 \%$. There is also a notable downward trend in the annual incidence of disease as measured by cases confirmed following on-farm reports of suspected disease: 403 such cases were recorded in 2002, compared to 99 in $2006^{1}$. However, these latter statistics have to be interpreted with caution as they may be influenced by whether or not farmers report all suspect cases.

The reduction in the detection rates for scrapie indicated by the various surveillance routes are encouraging, but further epidemiological analysis is required to determine to what extent the trend can be attributed to combined effects of the RGS and/or the control measures that have been targeted at known affected flocks.

Whilst addressing concerns over classical scrapie, large-scale industry-wide selection of animals on PrP genotype, raises many important issues, including those that cloud the industry perception of the breeding scheme and which may impede voluntary uptake. These issues include possible impacts on other performance traits, effects on inbreeding and genetic diversity in commercial populations, the prevalence of other forms of scrapie and the actual rate at which risks of classical scrapie are predicted to decline. However, most of these issues have been recognised by both industry and scientists, and are being addressed in parallel with breeding programmes based on PrP genotype.

\section{EFFECT OF PrP SELECTION ON OTHER PERFORMANCE TRAITS}

There are a number of ways in which selection on PrP genotype may impact upon other commercially important performance traits. For example, there may be a direct impact of the PrP gene on other traits of interest, such as lamb survival or growth rate, or aspects of maternal performance. Secondly, there may well be genes influencing performance traits that

\footnotetext{
${ }^{1}$ Del Rio Vilas V.J., Personal communication.
} 
are genetically linked with the PrP on chromosome 13 , i.e. in close vicinity to the PrP locus. Therefore specific alleles at these genes will tend to be inherited as a unit along with the PrP alleles. This can be disadvantageous if the linkage phase is such that the favourable alleles are inherited along with undesirable PrP alleles, but it can be advantageous if the linkage phase is reversed and the favourable performance trait alleles are inherited with favourable PrP alleles. Linkage phase can be expected to differ between populations, even between families in some instances, so that associations that are beneficial in one population may be deleterious in another. A third way that PrP selection can impact on performance traits is simply through the opportunity cost of diverting selection pressure away from traits that are normally selected on. In this case, a decline in performance traits is not expected; rather, the rate of gain will be reduced.

Associations between PrP genotype and performance are covered in detail by Sweeney and Hanrahan [37]. As a general summary, although some associations have been observed between PrP genotype and performance traits, these tend not to be strong, nor are they consistent between populations. Also, there is no discernable tendency for published associations between scrapie-resistant PrP alleles and performance traits to be adverse. From these published studies it is not generally possible to distinguish between direct (pleiotropic) effects of PrP genotype and effects due to linkage. For example, Moore et al. ${ }^{2}$ compared effects of PrP genotype on lamb performance traits in three major British Hill breeds, the Welsh Mountain, North Country Cheviot, and Scottish Blackface. Breed-specific effects that were seen included (i) $0.38 \mathrm{~kg}$ higher birth weights in North Country Cheviot AHQ/AHQ lambs relative to other genotypes $(p<0.01)$, (ii) $0.5 \mathrm{~kg}$ higher 8 week weights in VRQ heterozygous Scottish Blackface lambs relative to non-carriers $(p<0.01)$ and (iii) a decrease in ultrasonic scanning weight associ-

\footnotetext{
${ }^{2}$ Moore R.C., Boulton K., Bishop S.C., Associations of $\operatorname{PrP}$ genotype with lamb production traits in three commercial hill sheep breeds (manuscript in preparation).
}

ated with the ARR allele in Welsh Mountain lambs $(p<0.001)$. The absence of a common effect across the three breeds suggests that the associations are probably due to breed specific alleles of neighbouring genes in linkage disequilibrium with the PrP locus. However, chance associations, i.e. false positives, can not be ruled out.

Perhaps the only biologically explicable effect that has been detected to date in British studies, is an association between PrP genotype and coat colour patterns in primitive British breeds ${ }^{3}$. This can readily be explained by the Agouti locus which has a major influence on coat colour and patterns; this locus is closely linked to the PrP gene on chromosome 13. Therefore, it may be hypothesized that the apparent PrP coat colour effects are due to linkage disequilibrium between specific agouti and PrP alleles.

Opportunity cost effects associated with selection on PrP genotype have been explored in some detail by Man et al. [24]. In these simulation studies, it was assumed that flocks that were selecting on PrP genotype were already a part of a breeding programme in which intensive selection was being performed on a performance trait. Diverting selection pressure towards PrP genotype may well result in a decrease in the genetic progress made in other traits, although the impact is probably somewhat less than expected by many. The losses in genetic progress are a function of overall population size, PrP allele frequencies and selection strategies. If selection is against the VRQ allele only, then almost no impact is seen on genetic progress in other traits. An impact is seen if selection is for the ARR allele, but only when this allele is at a low frequency. For example, selection aimed at fixing the ARR allele in 15 years, in a population typical of a mainstream British Terminal sire breed, was predicted to result in a loss of ca. 2 years in genetic progress in performance traits, with most of this loss occurring early in the selection pro-

\footnotetext{
${ }^{3}$ Bell L., Goodman T., Martin J.H. et al., Survey of scrapie PrP genotype results and their relationships with coat colour and hornedness in selected UK rare sheep breeds, Proceedings of the British Society of Animal Science, 2004, p. 124.
} 
cess when most of the selection pressure was placed on increasing ARR frequency. This result was for a starting ARR frequency of 0.3 , if it was higher, then the reductions in gain in performance traits were less. In most mainstream British breeds ARR allele frequencies are now relatively high, and in some cases ARR is the most common allele. It should be noted that although Man et al. [24] considered the case of performance traits, the same arguments would apply if breeders were selecting for other attributes such as breed type characteristics.

\section{EFFECT OF PrP SELECTION ON INBREEDING AND GENETIC DIVERSITY}

Possible impacts on inbreeding and genetic diversity, i.e. increased inbreeding, population bottlenecks and decreased diversity, are often cited as a possible negative consequence of intense selection on PrP genotype. Again, consideration of precisely what PrP-based selection does is required. At the genetic level, selection is being performed on a genotype at one location on one autosome, out of 26 autosomes. Therefore, a marked reduction in genetic diversity can be expected at the PrP locus and in the immediate vicinity of this locus, but not elsewhere in the genome. At the population level, a decreased effective population size, and ultimately an increased rate of inbreeding, may possibly be expected if the PrP selection strategy forces the breeders to use fewer sire families than they would otherwise have done, or if similar total number of sires are used, but a small number (e.g. ARR/ARR rams) are used to a much greater extent than rams of other genotypes.

Possible effects of PrP selection on genetic diversity and/or inbreeding have been assessed using actual data arising from PrPbased selection programmes ${ }^{4}$ and also from simulation studies [24]. Palhière et al. studied

\footnotetext{
${ }^{4}$ Palhière I., Brochard M. et al., Did the selection for scrapie resistance impact genetic variability? Preliminary results on four French sheep breeds, 8th World Congress on Genetics Applied to Livestock Production, 2006, Communication 31-10.
}

genetic marker and geneological data arising from large scale French breeding programmes based on PrP genotype. Specifically, she assessed genetic marker heterozygosity on five microsatellite markers linked to PrP on chromosome 13, and 24 markers elsewhere in the genome. Further, using pedigree data she estimated the effects on population structure and dynamics. In terms of genetic marker properties, a large impact was seen on heterozygosity and the number of alleles present for markers linked to PrP, but only trivial impacts elsewhere in the genome. The population dynamics, as assessed by coefficients of relationship between individual animals, and the number of ancestors contributing $50 \%$ of the genes were largely unchanged. In other words, intense selection on PrP genotype did not markedly change genetic diversity or expected inbreeding rates. Given that selection on PrP genotype must focus selection on a small number of families that otherwise might not have been selected, why does it not therefore lead to potential inbreeding problems? The answer lies in the fact that directed selection for performance traits, in itself, is already a source of potential inbreeding. Redirecting some selection towards PrP genotype is, at worst, simply substituting one form of inbreeding for another. It may even be beneficial if it forces the breeder to use a wider range of families than would otherwise have been used.

These conclusions were reinforced by the modelling studies of Man et al. [24]. As described above, these studies investigated the dynamics of partial PrP-based selection in an ongoing breeding programme. In addition to having only small impacts on rates of gain on performance traits, it was found that rates of inbreeding were either the same as, or lower than, situations where selection was on performance traits. Thus it may be concluded that provided effective population sizes are not small, and efforts are made to avoid the mating of close relatives, then selection on PrP genotype will not have a deleterious impact on inbreeding. This assumes that breeders were previously positively selecting for improved animals, and not simply seeking to minimize inbreeding. 
These conclusions differ in situations where population sizes are very small, for example in rare or endangered breeds where the effective population size is less than, e.g., 50 animals. In this case there may be very few animals containing desirable PrP genotypes, and selective breeding on PrP may well cause unacceptable population bottlenecks. In this case, selection based on PrP genotype may not be a feasible option.

\section{RISKS OF SCRAPIE EPIDEMICS}

The ultimate aim of the PrP breeding programme is to reduce the incidence of classical scrapie, i.e. the risk that an epidemic occurs and the severity of the epidemic should it occur. Given the presence of the infectious agent in some flocks, clearly these risks are a function of the genotype profile of the population and the relative susceptibilities of the individual genotypes in the population, along with other non-genetic risk factors. Relative risks of classical scrapie for different genotypes may be deduced from data on cases of scrapie observed in commercial sheep, along with knowledge of the genotype distribution of the sheep populations from which these cases were reported. For example, Baylis et al. [3] reported an analysis of population-wide classical scrapie prevalence data and deduced the expected number of cases per million sheep. The ARQ/ARQ genotype may be used as the baseline, and 37 cases per million were reported for this genotype. Other notable results were zero case per million for ARR/ARR sheep, and 225, 405, and 545 cases per million for ARQ/VRQ, ARH/VRQ, and VRQ/VRQ genotypes, respectively. All other genotypes had fewer than ten cases per million. Tongue et al. [38] performed a similar analysis, expressing relative risks as odd ratios, and came to broadly similar conclusions.

The data from Baylis et al. [3] were then used in modelling studies [18], which assume transmission of infection through direct contact, to assess the impact of selection on PrP genotype on scrapie prevalence and incidence. Such selection strategies were indeed predicted to be successful in reducing the prevalence and incidence of disease, though there was likely to be a delay of several years between the implementation of a breeding programme and the reduction in incidence. As may be expected from the high relative risks incurred by having the VRQ allele, the major factor reducing scrapie risks was the elimination of the VRQ allele.

Several cautions should be applied to these results. Firstly, the results presented by Gubbins and Roden [18] are average or expected results. In reality, there will be variability of outcomes, with different outcomes arising in different flocks by chance alone. Detailed simulations which incorporate the stochastic nature of likely epidemic risks have been undertaken by Man $^{5}$ et al. These results show that even in cases where the population-wide prevalence of classical scrapie may be very low, often there are individual flocks where risks remain high. Therefore, it is very difficult to guarantee that PrP-based selection will eliminate classical scrapie from the national population. Secondly, these results assume transmission by direct contact; therefore elimination of infectious animals will eliminate the transmission of classical scrapie. This may not be the case if there are environmental reservoirs of infection. Such reservoirs may act as a source of infection into the future, and hence prolong the risks of scrapie. Thirdly, transmission was estimated as a function of the relative risk of classical scrapie, in turn estimated from the number of cases of clinical scrapie. This does not allow for the possibility that "resistant" animals may in fact be subclinically infected animals that are nevertheless transmitting infection. These latter two caveats would benefit from investigation through mathematical modelling studies. Although it is likely that their impacts on classical scrapie risks will be small, in relation to the major effect of eliminating the VRQ allele, they should nevertheless be investigated.

\section{EFFECTS ON OTHER FORMS OF SCRAPIE}

This paper has concentrated so far on PrP genotype in relation to classical scrapie. However, other forms of scrapie also exist. For

\footnotetext{
${ }^{5}$ Manuscript in preparation.
} 
example, a new type of scrapie, currently described as atypical scrapie, has been observed in many countries and its PrP genetics are different from those of classical scrapie $[1,4$, 23, 27, 32]. A recently described PrP polymorphism on the ARQ allele at codon $141(\mathrm{~L} / \mathrm{F})$, plays a major role in susceptibility and the animals most at risk are of genotypes AHQ/AHQ, $\mathrm{AF}_{141} \mathrm{RQ} / \mathrm{AF}_{141} \mathrm{RQ}$ and $\mathrm{AF}_{141} \mathrm{RQ} / \mathrm{AHQ}$. In contrast to classical scrapie, ARR/ARR sheep are susceptible to atypical scrapie, although often it remains a subclinical disease with minimal $\mathrm{PrP}^{\mathrm{Sc}}$ accumulation. Further, the VRQ allele does not incur extreme susceptibility to atypical scrapie as it does for classical scrapie. Although it is clearly evident that selection on PrP genotype, i.e. eliminating the VRQ allele and increasing the ARR frequency, will not be successful against atypical scrapie, calculations based on the relative risks of different genotypes suggest that this selection will be neutral regarding atypical scrapie (Baylis et al., personal communication). In other words, it will neither increase nor decrease risks of atypical scrapie.

More generally, concern arises from the observations on atypical scrapie along with numerous mouse studies that there may not be a universally scrapie resistant PrP genotype. Rather, it is widely believed that the outcome of an exposure depends upon the specific pairing of TSE strain and host PrP genotype. This effect can be substantial: in some circumstances a genotype considered resistant to one strain can be highly susceptible to another. This is important because substantial strain variation occurs in mouse scrapie, and it has also been demonstrated in surveys of field scrapie in sheep [8].

Lessons can be taken from the mouse where incubation period is also governed by TSE strain [13], as well as PrP genotype, as illustrated by large differences in incubation time (range ca. 150-460 days) within a single strain of inbred PrP A encoding mice challenged with different TSE strains [7].Further, challenge of PrP A encoding C57BL mice with mouse adapted scrapie strain Me7 results in incubation periods of ca. 160 days whilst identical challenges of PrP B encod- ing mice have longer incubation periods, ca. 360 days. Conversely, following challenge of the same mouse strains with mouse adapted sheep scrapie strain 22A the incubation time ranking is reversed such that PrP B mice may develop scrapie at ca. 190 days whilst PrP A mice take up to 480 days to develop clinical scrapie $[6,7]$. Thus, high or low susceptibility to TSE is not a fixed property of any given PrP genotype but the outcome of a specific pairing of host PrP genotype and TSE strain properties.

The concern arising from these findings is that whilst classical scrapie is currently the predominant strain present in populations containing large numbers of ARQ alleles accompanied by a low but significant proportion of VRQ alleles, this need not be the case in the future. There is a possible risk that as the sheep population genotype profile changes, then new forms of scrapie may arise that are adapted to the new predominant genotypes. This could potentially undermine current PrP selection strategies and is clearly a topic for further study, particularly as the likely prevalence and infectivity of non-classical forms of scrapie are unknown. In this regard, the detection of $\mathrm{PrP}^{\mathrm{Sc}}$ within the CNS of clinically normal young adult ARR/ARR sheep diagnosed with atypical scrapie should be noted [14]. Furthermore, the recent preliminary observation of two ARR/ARR sheep with clinical scrapie that is readily transmissible to mice [17], supports the view that there may be no ovine PrP genotype with absolute resistance to TSE. Long standing transgenic studies in scrapie infected mice [34] would predict that prions derived from subclinical or clinical infections of ARR/ARR sheep may be transmissible to healthy sheep of the same PrP genotype. If this is the case in practice, then care must be taken to avoid unintentionally creating populations with only one PrP genotype, even if it is ARR/ARR. Our current understanding from large scale surveillance is that ARR/ARR clinical scrapie is a very rare phenomenon, however the above arguments underline the need for the continued surveillance and the vigorous application of scrapie control measures, both genetic and non-genetic. 


\section{CONCLUSION}

The National Scrapie Plan for Great Britain has been a successful exercise and, along with similar programmes in other European countries, it demonstrates that industry-wide selection on PrP genotype is feasible. The voluntary Ram Genotyping Scheme has attracted participation from the majority of ram producers. With the help of the genotyping and selective culling programmes applied to affected flocks, it appears that scrapie is being brought under control. However, this conclusion should be reassessed at a future date when sufficient time has elapsed for the changes in PrP genotype profile to penetrate widely throughout the sheep industry and impact on scrapie risks.

The potential dangers of drastically reducing PrP genetic diversity in the national sheep flock have been widely recognized. Primarily these risks are associated with novel TSE agents that are as yet unknown but may emerge in the future, as discussed above, and the possible loss of favourable attributes of a breed either through linkage or pleiotropic effects when selecting on a specific PrP alleles. To guard against this risk, semen gene banks have been proposed and designed [31], and now implemented in Great Britain and Northern Ireland, to provide the potential for future reintroduction of removed alleles or genotypes. Further, these semen banks have been designed accounting for the specific population dynamics and demographics of each sector of the UK sheep industry.

These semen banks are particularly important in the potential case of the evolution of new forms of scrapie, in response to changes in the host population genotype profile. However, such arguments also warn against taking a population towards homogeneity, i.e. all animals having the same PrP genotype, as such a population would be particularly susceptible to a form of scrapie adapted to this genotype, and it would no longer be possible to select for resistance using PrP genotype. In fact, this is a generalization of the potential disease risks incurred in genetically homogenous populations [35]. Therefore, a suitable endpoint for a breeding programme based on PrP genotype is one where currently susceptible alleles are at a low frequency, but a good mix remains of other alleles.

One last means of guarding against future risks whilst increasing the resistance of the population to classical scrapie is to identify and utilize genes other than $\operatorname{PrP}$ that contribute to scrapie resistance. Evidence for such effects now exists in the mouse $[21,22,25,26,36]$, cattle $[19,41]$, and sheep $[12]^{6}$. Identification of the genes underlying this genetic variation in TSE resistance may allow selection for scrapie resistance that is effective against a wide variety of scrapie strains. This is an area urgently requiring further research.

Acknowledgements. S.C. Bishop and R.C. Moore wish to thank the Biotechnology and Biological Sciences Research Council (BBSRC) and the Department for Environment, Food and Rural Affairs (DEFRA) for financial support.

\section{REFERENCES}

[1] Arsac J.N., Andréoletti O., Bilheude J.M., Lacroux C., Benestad S.L., Baron T., Similar biochemical signatures and prion protein genotypes in atypical scrapie and Nor98 cases, France and Norway, Emerging Infect. Dis. (2007) 13:58-65.

[2] Arnold M., Meek C., Webb C.R., Hoinville L., Assessing the efficacy of a ram genotyping programme to reduce susceptibility to scrapie in Great Britain, Prev. Vet. Med. (2002) 56:227-249.

[3] Baylis M., Chihota C., Stevenson E., Goldmann W., Smith A., Sivam K., Tongue S., Gravenor M.B., Risk of scrapie in British sheep of different prion protein genotype, J. Gen. Virol. (2004) 85:2735-2740.

[4] Baylis M., McIntyre M., Transmissible spongiform encephalopathies: scrapie control under new strain, Nature (2004) 432:810-811.

[5] Benestad S.L., Arsac J.N., Goldmann W., Nöremark M., Atypical/Nor98 scrapie: properties of the agent, genetics, and epidemiology, Vet. Res. (2008) 39:19.

[6] Bruce M.E., McConnell I., Fraser H., Dickinson A.G., The disease characteristics of different strains of scrapie in sinc congenic mouse lines - implications for the nature of the agent and host control of pathogenesis, J. Gen. Virol. (1991) 72:595-603.

\footnotetext{
${ }^{6}$ Moreno C.R., Lantier I. et al., Transposition to sheep of mouse quantitative trait loci (QTL) influencing susceptibility to prion diseases, Proceedings of the 7th World Congress on Genetics Applied to Livestock Production, 2002, Communication 13-21.
} 
[7] Bruce M.E., Chree A., McConnell I., Wells G.A.H., Transmission of bovine spongiform encephalopathy and scrapie to mice: strain variation and the species barrier, Philos. Trans. R. Soc. Lond., B, Biol. Sci. (1994) 343:405-411.

[8] Bruce M.E., Boyle A., Cousens S., McConnell I., Foster J., Goldmann W., Fraser H., Strain characterization of natural sheep scrapie and comparison with BSE, J. Gen. Virol. (2002) 83:695-704.

[9] Davies D.C., Kimberlin R.H., Selection of Swaledale sheep of reduced susceptibility to experimental scrapie, Vet. Rec. (1985) 116:211-214.

[10] Dawson M., Genotyping service for Swaledale sheep, Vet. Rec. (1994) 134:583-584.

[11] Dawson M., Hoinville L.J., Hosie B.D., Hunter N., Guidance on the use of PrP genotyping as an aid to the control of clinical scrapie, Vet. Rec. (1998) 142:623-625.

[12] Diaz C., Vitezica Z.G., Rupp R., Andréoletti O., Elsen J.M., Polygenic variation and transmission factors involved in the resistance/susceptibility to scrapie in a Romanov flock, J. Gen. Virol. (2005) 86:849-857.

[13] Dickinson A.G., Fraser H., Scrapie pathogenesis in inbred mice: an assessment of host control and response involving many strains of agent, in: Slow virus infections of the central nervous system, Ter Meulen V., Katz M. (Eds.), Springer, New-York, USA, 1977, pp. 3-14.

[14] Everest S.J., Thorne L., Barnicle D.A., Edwards J.C., Ellitt H., Jackman R., Hope J., Atypical prion protein in sheep brain collected during the British scrapie surveillance programme, J. Gen. Virol. (2006) 87:471477.

[15] Foster J.D., Hope J., Fraser H., Transmission of bovine spongiform encephalopathy to sheep and goats, Vet. Rec. (1993) 133:339-341.

[16] Gonzales L., Jeffrey M., Siso S., Martin S., Bellworthy S.J., Stack M.J., Chaplin M.J., Davis L., Dalgleish M.P., Reid H.W., Diagnosis of preclinical scrapie in samples of rectal mucosa, Vet. Rec. (2005) 156:846-847.

[17] Groschup M.H., Lacroux C., Buschmann A., Luhken G., Mathey J., Eiden M., Lugan S., Hoffmann C., Espinosa J.C., Baron T.,Torres J.M., Erhardt G., Andréoletti O., Classic scrapie in sheep with the ARR/ARR prion genotype in Germany and France, Emerging Infect. Dis. (2007) 13:1201-1207.

[18] Gubbins S., Roden J.A., Breeding programmes for TSE resistance in British sheep. II. Assessing the impact on the prevalence and incidence of scrapie, Prev. Vet. Med. (2006) 73:17-31.

[19] Hernandez-Sanchez J., Waddington D., Wiener P., Haley C.S., Williams J.L., Genome-wide search for markers associated with bovine spongiform encephalopathy, Mamm. Genome (2002) 13:164-168.

[20] Hunter N., Goldmann W., Foster J.D., Cairns D., Smith G., Natural scrapie and PrP genotype: case control studies in British sheep, Vet. Rec. (1997) 141:137140.
[21] Lloyd S.E., Onwuazor O.N., Beck J.A., Mallinson G., Farrall M., Targonski P., Collinge J., Fisher E.M.C., Identification of multiple quantitative trait loci linked to prion disease incubation period in mice, Proc. Natl. Acad. Sci. USA (2001) 98:6279-6283.

[22] Lloyd S.E., Uphill J.B., Targonski P.V., Fisher E.M.C., Collinge J., Identification of genetic loci affecting mouse-adapted bovine spongiform encephalopathy incubation time in mice, Neurogenetics (2002) 4:77-81.

[23] Lühken G., Buschmann A., Brandt H., Eiden M., Groschup M.H., Erhardt G., Epidemiological and genetical differences between classical and atypical scrapie cases, Vet. Res. (2007) 38:65-80.

[24] Man N.W.Y., Lewis R.M., Boulton K., Villanueva B., Predicting the consequences of selecting on PrP genotypes on PrP frequencies, performance and inbreeding in commercial meat sheep populations, Genet. Sel. Evol. (2007) 39:711-729.

[25] Manolakou K., Beaton J., McConnell I., Farquar C., Manson J., Hastie N.D., Bruce M., Jackson I.J., Genetic and environmental factors modify bovine spongiform encephalopathy incubation period in mice, Proc. Natl. Acad. Sci. USA (2001) 98:7402-7407.

[26] Moreno C.R., Lantier F., Lantier I., Sarradin P., Elsen J.M., Detection of new quantitative trait loci for susceptibility to transmissible spongiform encephalopathies in mice, Genetics (2003) 165:20852091.

[27] Moum T., Olsaker I., Hopp P., Moldal T., Valheim M., Moum T., Benestad S.L., Polymorphisms at codon 141 and 154 in the ovine prion protein gene are associated with scrapie Nor98 cases, J. Gen. Virol. (2005) 86:231-235.

[28] O’Rourke K.I., Baszler T.V., Besser T.E., Miller J.M., Cutlip R.C., Wells G.A.H., Ryder S.J., Parish S.M., Hamir A.N., Cockett N.E., Jenny A., Knowles D.P., Preclinical diagnosis of scrapie by immunohistochemistry of third eyelid lymphoid tissue, J. Clin. Microbiol. (2000) 38:3254-3259.

[29] Parry H.B., in: Scrapie disease in sheep, Oppenheimer D.R. (Ed.), Academic Press, London, UK, 1983, pp. 31-59.

[30] Pollott G.E., Stone D.G., The breeding structure of the British sheep industry, 2003, Defra publications, London, UK, 2004.

[31] Roughsedge T., Villanueva B., Woolliams J.A., Determining the relationship between restorative potential and size of a gene bank to alleviate the risks inherent in a scrapie eradication breeding programme, Livest. Sci. (2006)100: 231-241.

[32] Saunders G.C., Cawthraw S., Mountjoy S.J., Hope J., Windl O., PrP genotypes of atypical scrapie cases in Great Britain, J. Gen. Virol. (2006) 87:31413149.

[33] Schreuder B.E.C., van Keulen L.J.M., Vromans M.E.W., Langeveld J.P.M., Smits M.A., Tonsillar biopsy and $\mathrm{PrP}^{\mathrm{Sc}}$ detection in the preclinical diagnosis of scrapie, Vet. Rec. (1998) 142:564-568. 
[34] Scott M., Foster D., Mirenda C., Serban D., Coufal F., Wälchli M., Torchia M., Groth D., Carlson G., DeArmond S.J., Westaway D., Prusiner S.B., Transgenic mice expressing hamster prion protein produce species-specific scrapie infectivity and amyloid plaques, Cell (1989) 59:847-857.

[35] Springbett A.J., MacKenzie K., Woolliams J.A., Bishop S.C., The contribution of genetic diversity to the spread of infectious diseases in livestock populations, Genetics (2003) 165:1465-1474.

[36] Stephenson D.A., Chiotti K., Ebeling C., Groth D., DeArmond S.J., Prusiner S.B., Carlson G.A., Quantitative trait loci affecting prion incubation time in mice, Genomics (2000) 69:47-53.

[37] Sweeney T., Hanrahan J.P., The evidence of associations between prion protein genotype and production, reproduction and health traits in sheep, Vet. Res. (2008) 39:28, in press.
[38] Tongue S.C., Pfeiffer D.U., Warner R., Elliott H., Vilas V.D., Estimation of the relative risk of developing clinical scrapie: the role of prion protein (PrP) genotype and selection bias, Vet. Rec. (2006)158:43-50.

[39] Warner R.G., Morris D., Dawson M., PrP genotype progression in flocks participating in the National Scrapie Plan for Great Britain, Vet. Rec. (2006) 159:473-479.

[40] Will R.G., Ironside J.W., Zeidler M., Cousens S.N., Estibeiro K., Alperovitch A., Poser S., Pocchiari M., Hofman A., Smith P.G., A new variant of Creutzfeldt-Jakob disease in the UK, Lancet (1996) 347:921-925.

[41] Zhang C., De Koning D.J., Hernandez-Sanchez J., Haley C.S., Williams J.L., Wiener P., Mapping of multiple quantitative trait loci affecting bovine spongiform encephalopathy, Genetics (2004) 167:1863-1872. 